\title{
The profiles of lipid peroxidation and anti-oxidant activities in gestational diabetes mellitus \& normal pregnancies in Nigerian population
}

\author{
Adetunji O. Adeniji ${ }^{1}$, Dolapo P. Oparinde ${ }^{2 \#}$ \\ ${ }^{1}$ Department of Obstetrics \& Gynaecology, College of Health Sciences, Ladoke Akintola University of Technology, Ogbomoso, \\ Nigeria \\ ${ }^{2}$ Department of Chemical Pathology, College of Health Sciences, Ladoke Akintola University of Technology, Ogbomoso, Nigeria \\ Email: "piusdollars@yahoo.com
}

Received 14 June 2013; revised 12 July 2013; accepted 19 July 2013

Copyright (C) 2013 Adetunji O. Adeniji, Dolapo P. Oparinde. This is an open access article distributed under the Creative Commons Attribution License, which permits unrestricted use, distribution, and reproduction in any medium, provided the original work is properly cited.

\begin{abstract}
Purpose: To compare the Lipid peroxidation and Total antioxidant status in women with gestational diabetes mellitus and normal pregnancy in our environment. Materials \& Methods: This was a 2-year, cross sectional, case control study of 25 gestational diabetes mellitus (GDM) and 75 matched normal pregnant women at Ladoke Akintola University of Technology (LAUTECH) Teaching Hospital, Osogbo, Nigeria. The study protocol was reviewed and approved by our Institutional Ethical Review Committee and all participants gave their consents. The fasting serum of recruited patients were analysed for lipid peroxidetion product malondialdehyde(MDA), based on MDA reaction with thiobarbituric acid (TBA), with rapid, sensitive and specific Thiobarbituric Acid (TBA) assay, while the Total Anti-oxidant status (TAS) was determined using the capacity of the biological fluids to inhibit the production of thiobarbituric acid reactive substances (TBARS) from sodium benzoate under the influence of the free oxygen radicals derived from Fenton's reaction. Results: The mean serum MDA was significantly higher in the GDM group $(3.64 \mathrm{nmol} / \mathrm{mL})$ than the value recorded $(1.33 \mathrm{nmol} /$ $\mathbf{m L})$ in the control group $(P<0.0001)$. Conversely, significantly lower mean TAS $(0.34 \mathrm{nmol} / \mathrm{mL}$ vs. 0.66 $\mathrm{nmol} / \mathrm{mL})$ was noticed in the GDM group $(P<\mathbf{0 . 0 0 0 1})$. Conclusion: The study showed significant increase in lipid peroxidation and failure of compensatory antioxidant functions in GDM Nigerian women.
\end{abstract}

\footnotetext{
${ }^{*}$ Conflict of interest: We declare that no funding/grant was received for this study and no conflict of interest or commercial relationship. ${ }^{\#}$ Corresponding author.
}

Keywords: Lipid Peroxidation; Anti-Oxidant Status; Gestational Diabetes Mellitus; Malondialdehyde

\section{INTRODUCTION}

The World Health Organisation defined gestational diabetes mellitus (GDM) as carbohydrate intolerance resulting in hyperglycaemia of variable severity with onset or first recognition that usually during the second half of pregnancy. It affects $3 \%-10 \%$ of pregnancies, depending on the population studied [1]. This definition applies irrespective of whether or not insulin is used for treatment. In Nigerian population a prevalence of $1.7 \%$ had been reported for diabetes in pregnancy, with GDM accounting for $61 \%$ of them [2]. Pregnancy is a physiological condition characterised by various potentially diabetogenic hormonal changes due to increase in glucocorticoids, human placental lactogen and oestrogens. This can result in insulin resistance and it may be the first life challenge of a woman's ability to respond to a physiological stress and to detect those at greater risk of developing diabetes in the future. Women with GDM are at 3-fold increased risk of developing Type 2 diabetes mellitus later in life [3] and thus much attention has been focussed on the interlink between these two metabolic conditions. Whether Type 2 diabetes mellitus is a continuum of GDM is yet to be fully established. However, both diseases share similar pathophysiology and it is generally known that the higher the prevalence of type 2 diabetes in the population, the higher that of GDM. Oxidative stress which is a general term used to describe the steady state level of oxidative damage to cell, tissue or organ, caused by the elevated reactive oxygen species (ROS) [4], has been associated with type 2 diabetes mel- 
litus, however, limited and inconsistent data are available on the involvement of oxidative stress in GDM.

Lipid peroxidation, formation of free radicals and reactive oxygen species are normal occurrences in pregnancy, but these happen at low level in normal pregnancy, with its peak in the second trimester of pregnancy. In normal pregnancy, the balance between lipid peroxidations, free radicals (pro-oxidants) and the antioxidants formations are well maintained in order to minimize cellular damage [5,6]. When the balance is disrupted towards an overabundance of ROS, oxidative stress occurs.

Hyperglycaemia causes oxidative stress due to increased production of mitochondrial ROS, excessive nonenzymatic formation of glycosylated proteins, associated alterations in the uptake of low-density lipoproteins and glucose autoxidation [7,8]. Elevated FFA can also cause oxidative stress due to increased mitochondrial uncoupling and $\beta$-oxidation, leading to the increased production of $\operatorname{ROS}[9,10]$.

Reports in respect of pro-oxidant and anti-oxidant status in GDM have been inconsistent and controversial. While some studies had reported significant increase in lipid peroxidation activities in this condition, when compared with normal pregnancy [11-14], others found no evidence of greater lipid peroxidation and in fact concluded that total antioxidant capacity was similar in GDM women when compared with normal pregnancy $[15,16]$.

In Nigeria, published information about the serum lipid peroxides and antioxidants status in gestational diabetes mellitus women in our environment is scarce. For this reason, this study has been undertaken to establish the serum malondialdehyde (MDA) and Total antioxidant status in women with GDM and compare with women of normal pregnancy in our environment.

\section{MATERIALS AND METHODS}

This was a cross sectional, case control study of pregnant patients at Ladoke Akintola University of Technology (LAUTECH) Teaching Hospital, Osogbo, Nigeria. The study was over a 2-year period (2008-2010) and the study protocol was reviewed and approved by our Institutional Ethical Review Committee. All participants gave informed consent to participate in the study.

The diagnosis of gestational diabetes mellitus and inclusion in the study were in accordance with the guidelines of American Diabetes Association criteria [3], which stipulated two or more results of the 100 gram oral glucose tolerance test (OGTT) glucose levelsto exceed the following cut-off values: fasting: $\geq 95 \mathrm{mg} / \mathrm{dl}(5.3$ $\mathrm{mmol} / \mathrm{L})$; one hour: $\geq 180 \mathrm{mg} / \mathrm{dl}(10.0 \mathrm{mmol} / \mathrm{L})$; two hours: $\geq 155 \mathrm{mg} / \mathrm{dl}(8.6 \mathrm{mmol} / \mathrm{L})$; three hours: $\geq 140$ $\mathrm{mg} / \mathrm{dl}$ (7.8 mmol/L). Patients' recruitment and samples collection for assessment of lipid peroxidation and total antioxidant status were collected same time as for fasting plasma glucose and only processed if diagnosis of GDM was confirmed. Other samples in which GDM was not established were matched along with other normal pregnant patients recruited and processed as controls. The exclusion criteria were women with pre-pregnancy diabetes mellitus, obesity, chronic hypertension, haemoglobinopathy, dyslipidaemia, multiple pregnancy, liver diseases, history of using antioxidant medications and patient's refusal of consent to participate in the study. All GDM patients were managed by their primary obstetricians, initially by diet control and when necessary with insulin.

A total of 100 pregnant women-25 gestational diabetes mellitus \& 75 normal pregnant patients (controls) -were recruited for the study. Each recruited GDM patient was matched with 3 normal pregnant patients, as controls, for age ( \pm 2 years), estimated gestational age $( \pm 1$ week) and parity.

Assay for Lipid peroxidation and Antioxidant status were done with ten millilitre of fasting peripheral venous blood collected into sterile plain bottle and centrifuged at $4000 \mathrm{rpm}$ for 10 minutes. The resultant serum was collected into fresh sterile, acid-washed, plain capped bottle and stored at $-20^{\circ} \mathrm{C}$ until analysis. Lipid peroxidation product, serum malondialdehyde (MDA) level, was assayed for using a rapid, sensitive and specific Thiobarbituric Acid assay [17], based on MDA reaction with thiobarbituric acid (TBA) and the Total Anti-oxidant status (TAS) was determined as by Koracevic et al. [18]. In Koracevic method, a standardised solution of Fe-EDTA complex reacts with hydrogen peroxide by a Fenton-type reaction, leading to the formation of hydroxyl radicals $(\cdot \mathrm{OH})$. These reactive oxygen species degrade benzoate, resulting in the release of TBARS. Antioxidants from the added sample of human fluid cause suppression of the production of TBARS. This reaction can be measured spectrophotometrically and the inhibition of colour development defined as the anti-oxidative activity.

\section{Statistics}

Data obtained was analysed with Statistical Package for Social Sciences version 16 (SPSS Inc, Chicago, IL, USA) for continuous variables as mean \pm standard deviation, Student t-test and confidence interval. Level of significance was set at $\mathrm{P} \leq 0.05$.

\section{RESULTS}

A total of 100 patients were recruited for study, of which 25 were women diagnosed with gestational diabetes mellitus and 75 were normal pregnant women (Controls). The women recruited as "controls" were matched at ratio of 3 for each woman with gestational diabetes mellitus 
for age ( \pm 2 years), estimated gestational age ( \pm 1 week) and parity. There were no significant differences in the mean weight, estimated gestational age and parity distribution in both group at recruitment. The mean age of patients recruited in the GDM group was significantly higher than in the control (35.65 years vs 33.26 years; $\mathrm{P}$ $<0.0001$ ), so also was the body mass index, which was extremely significantly different in the GDM group than control [Table 1]. The mean height of the participants was significantly lower $(\mathrm{P}=0.0005)$ in the GDM group than the control group (1.59 $\mathrm{m}$ vs $1.62 \mathrm{~m})$.

In Table 2, the mean serum MDA was significantly higher in the GDM group $(3.64 \pm 0.64 \mathrm{nmol} / \mathrm{mL})$ than the value recorded $(1.33 \pm 0.15 \mathrm{nmol} / \mathrm{mL})$ in the control group $(\mathrm{P}<0.0001)$. Conversely, significantly lower mean TAS of $0.34(0.10) \mathrm{nmol} / \mathrm{mL}$ was recorded in the GDM group, in contrast to mean value of $0.66(0.13)$ $\mathrm{nmol} / \mathrm{mL}$ in the control group $(\mathrm{P}<0.0001)$.

\section{DISCUSSION}

The findings from this study showed increased lipid peroxidation and reduced levels of total antioxidant status in GDM women above those in the normal pregnant women. These are similar to the conclusions from other studies [8,11-14]. However, some other studies had also reported that no significant difference existed in the lipid peroxidation and the antioxidant status between GDM patients and controls [15,16,19]. The findings from this study showed about 3 -fold increase in the lipid peroxidation activities and concurrently, 2-fold decrease in total anti-oxidant status in women with GDM, when compared with the normal pregnant women. These findings suggest a great tendency for enhanced and uncompensated free radical generation, which can lead to easier membrane damage in GDM patients and other adverse effects of oxidative stress. Primary effect of increased oxygen free radical in gestational diabetes is believed to alter prostaglandin biosynthesis which may be responseble for the development of diabetes related embryopathy [20]. Increased oxidative stress in pregnant women and possibly their fetuses has been associated with congenital malformations [21]. Inadequate antioxidant defense has been postulated as a prime factor in pathologic states of many premature infants [22]. However, this study was

Table 1. Socio-demographic factors of gestational diabetes mellitus women and the normal pregnant controls.

\begin{tabular}{|c|c|c|c|c|c|}
\hline PARAMETERS & $\begin{array}{c}\text { GESTATIONAL } \\
\text { DIABETES MELLITUS } \\
\text { PATIENTS } \\
\mathbf{n}=25 \\
\text { mean (SD) }\end{array}$ & $\begin{array}{c}\text { CONTROL } \\
n=75 \\
\text { mean (SD) }\end{array}$ & $\mathbf{t}$ & p-value & 95\% C.I \\
\hline Age (Years) & $35.65(2.29)$ & $33.26(2.32)$ & 4.4749 & $<0.0001$ & $1.3301,3.4499$ \\
\hline EGA (Weeks) & $28.64(6.45)$ & $28.58(6.39)$ & 0.0406 & 0.9677 & $-2.8753,2.9953$ \\
\hline Weight (kg) & $74.40(8.16)$ & $73.35(5.42)$ & 0.7329 & 0.4654 & $-1.7932,3.8932$ \\
\hline Height (m) & $1.59(0.02)$ & $1.62(0.04)$ & 3.5944 & 0.0005 & $-0.0466,-0.0134$ \\
\hline BMI $\left(\mathrm{Kg} / \mathrm{m}^{2}\right)$ & $29.43(2.34)$ & $27.94(2.26)$ & 2.8300 & 0.0056 & $0.4452,2.5348$ \\
\hline Fasting Plasma Glucose (mmol/1) & $105.21(10.18)$ & $72.23(15.21)$ & 10.0963 & $<0.0001$ & $26.4977,39.4623$ \\
\hline Plasma Glucose -2 hr (mmol/1) & $185.36(24.52)$ & $129.22(16.74)$ & 12.8328 & $<0.0001$ & $47.4585,64.8215$ \\
\hline Plasma Glucose $-3 \mathrm{hr}(\mathrm{mmol} / \mathrm{l})$ & $126.65(14.64)$ & $85.32(15.23)$ & 11.8616 & $<0.0001$ & $34.4154,48.2446$ \\
\hline
\end{tabular}

Key: BMI—Body Mass Index; EGA—Estimated Gestational Age.

Table 2. Comparison of serum malondialdehyde \& total antioxidant status in gestational diabetes mellitus women $\&$ normal pregnant controls.

\begin{tabular}{cccccc}
\hline FACTORS & $\begin{array}{c}\text { GESTATIONAL DIABETES } \\
\text { MELLITUS PATIENTS } \\
\mathbf{n}=\mathbf{2 5} \\
\text { mean (SD) }\end{array}$ & $\begin{array}{c}\text { CONTROL } \\
\mathbf{n}=\mathbf{7 5} \\
\text { mean (SD) }\end{array}$ & $\mathbf{t}$ & p-value & $\mathbf{9 5 \% \text { C.I }}$ \\
\hline MDA $(\mathrm{nmol} / \mathrm{mL})$ & $3.64(0.64)$ & $1.33(0.15)$ & 29.2054 & $<0.0001$ & $2.1530,2.4670$ \\
TAS $(\mathrm{nmol} / \mathrm{mL})$ & $0.34(0.10)$ & $0.66(0.13)$ & 11.2353 & $<0.0001$ & $-0.3765,-0.2635$ \\
\hline
\end{tabular}

Key: MDA—Malondialdehyde; TAS—Total Antioxidant Status. 
not designed to assess the fetal parameters.

If lipid peroxidation status in GDM has been controversial, the antioxidants parameters have not been less, with many conflicting reports in the literatures. While some Researchers had reported decline of various antioxidant agents $[8,13,23]$ when compared with normal pregnant women, others reports had claimed no change in antioxidants status of GDM patients $[15,20]$. However, our finding indicated a 2-fold decrease in total antioxidants status of GDM patients in our population. It may be reasoned therefore from our finding that addition of supplemental antioxidant nutrients, such as vitamins $\mathrm{C}, \mathrm{E}$, carotenoids, selenium and others may be beneficial in improving the TAS status in GDM patients. This however may require further investigations for possible justification in clinical practice.

Patients recruited for this study were matched for age, estimated gestational age and parity. These strict enrolment criteria were to eliminate the confounding effects of these socio-demographic factors. Despite these efforts, patients with GDM were significantly older and were heavier in weight, though of shorter heights. The impact of higher weight and shorter height reflected on the BMI of the GDM group, with the mean value of $29.43 \mathrm{Kg} / \mathrm{m}^{2}$, which is pre-obese by the World Health Organisation classification [24]. Reports in literature had associated maternal pre-pregnancy weight, as well as weight gain in pregnancy, especially in the first trimester as independent risk factors for GDM $[25,26]$. However, in this study, it was not possible to explore these factors because most patients were seen in pregnancy and mostly in advanced gestations. A structured pre-conception care for all mothers-to be would definitely be of benefit in properly assessing the influences of these socio-demographic factors.

\section{CONCLUSION}

This study demonstrated significant increase in lipid membrane damage activities (lipid peroxidation), as evidenced by the elevated serum MDA in GDM women and failure of compensatory antioxidant functions demonstrated by overall lower antioxidant capacity in this group of pregnant women. These further buttress a possible link between oxidative stress and GDM. Also, significant factors noted in these GDM patients were increased maternal age, body mass index and short stature. Further researches on possible effects of antioxidant supplements may be justified.

\section{ACKNOWLEDGEMENTS}

We are grateful to all our patients who consented to participate in this study and all our resident Doctors that assisted in the conduct of the study.

\section{REFERENCES}

[1] World Health Organization (1999) Definition, diagnosis and classification of diabetes mellitus and its complications. Geneva.

[2] Ozumba, B.C., Obi, S.N. and Oli, J.M. (2004) Diabetes mellitus in pregnancy in an African population. International Journal of Gynecology \& Obstetrics, 84, 114-119. doi:10.1016/S0020-7292(03)00210-8

[3] American Diabetes Association (2004) Gestational diabetes mellitus. Diabetes Care, 27, 588-590.

[4] Oxidative stress (2013) Wikipedia Foundation Inc. http://en.wikipedia.org/wiki/Oxidative_stress

[5] Wickens, D., Wilikins, M.H., Lunce, J., Ball, G. and Dormandy, T.L. (1998) Free radical oxidation products in normal and abnormal pregnancy. Annals of Clinical Biochemistry, 18, 158-162.

[6] Poranen, A.K., Ekblad, U., Uotila, P. and Ahotupa, M. (1996) Lipid peroxidation and antioxidants in normal and preeclamptic pregnancies. Placenta, 17, 401-405. doi:10.1016/S0143-4004(96)90021-1

[7] Brownlee, M. (2001) Biochemistry and molecular cell biology of diabetic complications. Nature, 414, 813-820. doi:10.1038/414813a

[8] Suhail, M., Patil, S., Khan, S. and Siddiqui, S. (2010) Antioxidant vitamins and lipoper oxidation in non-pregnant, pregnant, and gestational diabetic women: Erythrocytes osmotic fragility profiles. Journal of Clinical Medicine Research, 2, 266-273.

[9] King, G.L. and Loeken, M.R. (2004) Hyperglycemia-induced oxidative stress in diabetic complications. Histochemistry and Cell Biology, 122, 333-338. doi:10.1007/s00418-004-0678-9

[10] Wender-Ozegowska, E., Kozlik, J., Biczysko, R. and Ozegowski, S. (2004) Changes of oxidative stress parameters in diabetic pregnancy. Free Radical Research, 38, 795-803. doi:10.1080/10715760410001700479

[11] Carone, D., Loverro, G., Greco, P., Capuano, F. and Selvaggi, L. (1993) Lipid peroxidation products and antioxidant enzymes in red blood cells during normal and diabetic pregnancy. European Journal of Obstetrics \& Gynecology and Reproductive Biology, 51, 103-109. doi:10.1016/0028-2243(93)90021-4

[12] Chaudhari, L., Tandon, O. P., Vaney, N. and Agarwal, N. (2003) Lipid peroxidation and antioxidant enzymes in gestational diabetics. Indian Journal of Physiology and Pharmacology, 47, 441-446.

[13] Surapaneni, K.M. (2007) Oxidant-antioxidant status in gestational diabetes patients. Journal of Clinical and Diagnostic Research. 1, 235-238.

[14] Karacay, O., Sepici-Dincel, A., Karcaaltincaba, D., Sahin, D., Yalvaç, S., Akyol, M., Kandemir, O. and Altan, N.A. (2010) Quantitative evaluation of total antioxidant status and oxidative stress markers in preeclampsia and gestational diabetic patients in 24-36 weeks of gestation. Dia- 
betes Research and Clinical Practice, 89, 231-238. doi:10.1016/i.diabres.2010.04.015

[15] Bates, J.H., Young, I.S., Galway, L., Traub, A.I. and Hadden, D.R. (1997) Antioxidant status and lipid peroxidation in diabetic pregnancy. British Journal of Nutrition, 78, 523-532. doi:10.1079/BJN19970172

[16] Sobki, S.H., Al-Senaidy, A.M., Al-Shammari, T.A., Inam, S.S., Al-Gwiser, A.A. and Bukhari, S.A. (2004) Impact of gestational diabetes on lipid profiling and indices of oxidative stress in maternal and cord plasma. Saudi Medical Journal, 25, 876-880.

[17] Botsuglou, N.A. (1994) Rapid, sensitive and specific thiobarbituric acid method for measuring lipid peroxidetion in animal tissue food and feedstuff samples. Journal of Agricultural and Food Chemistry, 42,1993-1997

[18] Koracevic, D., Koracevic, G., Djordjevic, V., Andrejevic, S. and Cosic, V. (2001) Method for the measurement of antioxidant activity in human fluids. Journal of Clinical Pathology, 54, 356-361. doi:10.1136/jep.54.5.356

[19] Dey, P., Gupta, P., Acharya, N.K., Rao, S.N., et al. (2008) Antioxidants and lipid peroxidation in gestational diabetes-A preliminary study. Indian Journal of Physiology and Pharmacology, 52, 149-156.

[20] Sivan, E., Lee, Y.C., Wu Y.K. and Reece, E.A. (1997) Free radical scavenging enzyme in fetal dysmorphogenesis among offspring of diabetic rats. Teratology, 56, 343349.
doi:10.1002/(SICI)1096-9926(199712)56:6<343::AID-T ERA1>3.0.CO;2-X

[21] Bis-Gluchowska, M., Marciniak, B., Szpringer-Bogun, E., Rola, R., Leszczynsdka-Gorzelak, B. and Oleszczuk, J. (2001) Determination of antioxidative-peroxidative balance in the cord blood of newborns delivered to mothers with diabetes type G1. Ginekologia Polska, 72, 12551258.

[22] Saugstand, O.D. (1990) Oxygen toxicity in the neonatal period. Acta Paediatrica Scandinavica Journal, 67, 735739.

[23] Kharb, S. (2007) Ascorbic acid and uric acid levels in gestational diabetes mellitus. The Journal of Obstetrics \& Ganecology of India, 57, 401-402.

[24] WHO Expert Consultation (2004) Appropriate body-mass index for Asian populations and its implications for policy and intervention strategies. The Lancet, 363, 157-163.

[25] Hedderson, M.M., Gunderson, E.P. and Ferrara, A. (2010) Gestational weight gain and risk of gestational diabetes mellitus. Obstetrics \& Gynecology, 115, 597-604. doi:10.1097/AOG.0b013e3181cfce4f

[26] Horosz, E., Bomba-Opon, D.A., Szymanska, M. and Wielgos, M. (2013) Maternal weight gain in women with gestational diabetes mellitus. Journal of Perinatal Medicine, 1-6. [Epub ahead of print]

doi:10.1515/jpm-2012-0254 